\title{
VORTEX FORMATION IN INCOMPRESSIBLE AXISYMMETRIC FREE JETS
}

\author{
E. R. V. Del Rio, | ABSTRACT \\ E. R. Woiski, \\ J. L. Gasche, \\ Free jets have been utilized extensively in many industrial applications. In \\ general jet fluid discharging from a nozzle develops flow oscillations in the \\ shear layer. The oscillations will roll up to eventually become toroidal \\ S. S. Mansur, \\ and E. D. R. Vieira \\ vortices which increase in size with the axial distance from the nozzle. In \\ the present work, flow visualization as well as hot-film anemometry have \\ been employed in order to study incompressible axisymmetric free jet in \\ moderate Reynolds numbers up to 20,000. The injection of liquid dye or \\ micro particles associated with a laser sheet turns possible to visualize the \\ shear layers and, consequently, the vortex formation. Hot-film \\ measurements into the jet allow determining the flow velocity profile. Flow \\ visualization is a qualitative tool which a broad view of the flow topology. \\ On the other hand, hot-film anemometry is a precise quantitative tool but \\ measurement in only one location at a time. The association of flow \\ visualization and hot-film anemometry shows very adequate for free jet \\ studies. \\ CEP 15385-000, Ilha Solteira, SP, Brasil \\ delrio@dem.feis.unesp.br \\ Received: October 29, 2014 \\ Revised: November 30, 2014 \\ Accepted: December 30, 2014 \\ Keywords: flow visualization, free jet, vortex formation, hot-film anemometry
}

\section{NOMENCLATURE}

$C R$ contraction ratio

$D$ diameter, $\mathrm{m}$

$f$ frequency, $1 / \mathrm{s}$

St Strouhal number

Re Reynolds number

$u(r)$ longitudinal velocity, $\mathrm{m} / \mathrm{s}$

$r$ radial coordinate, $\mathrm{m}$

$U$ mean velocity, $\mathrm{m} / \mathrm{s}$

$x \quad$ longitudinal coordinate, $\mathrm{m}$

\section{Greek symbols}

$\lambda$ distance between two consecutive vortices, $\mathrm{m}$

$\delta \quad$ free jet width, $\mathrm{m}$

$v \quad$ fluid kinematic viscosity, $\mathrm{m}^{2} / \mathrm{s}$

$\theta$ momentum thickness, $\mathrm{m}$

\section{Subscripts}

in contraction inlet

out contraction exit (jet root)

$\theta \quad$ momentum thickness

\section{INTRODUCTION}

A jet is one of the oldest techniques of intensification of heat exchange processes. Cooling, heating, and drying by convective processes can be easily intensified by impinging jets. Jet flow engineering application have been encountered in textile manufacturing, electronic cooling, steam and gas turbines, ejectors, industrial furnaces, chemical process, pollutant discharge, mixing process and pneumatic systems. A wide variety of flow configurations is characteristic of jet flows. Jets can be classified by the flow regime (laminar, turbulent), by geometry (plane, axisymmetric), by mutual direction of the jet spreading and motion of the surrounding fluid (co, counter and cross flows) and by the interaction with the surrounding fluid and solid walls (no walls contact, or wall impinging jets). The work of Yarin (2012) displays a number of jet flow configurations and a detailed study of their nondimensional analysis for turbulent and laminar regime. Theoretical laminar analysis for several jets types are found in the works of Pai (1954) and Schlichting (1968).

An axisymmetric jet is produced whenever a fluid is ejected from a round nozzle. Several exit velocity profiles can be generated depending on the geometrical characteristics of the nozzle. For instance, if the contraction ratio of the nozzle is large enough the velocity distribution is more or less constant over the cross-section and is commonly denoted as a "top-hat" profile or a plug flow. Figure 1 shows schematically a round jet issuing from a nozzle with diameter $D_{\text {out }}$, and its evolution along the streamwise axis, $x$. The contraction ratio $(C R)$ can be defined as the ratio of the inlet contraction diameter $\left(D_{i n}\right)$ and the nozzle lip diameter $D_{\text {out }}$. Due to the high velocity gradients next to nozzle lip, an axisymmetric 
shear layer forms and develops between the jet and the much slower (or even quiescent) surrounding fluid. It is within this region that the velocity of the jet will gradually approach that of the ambient fluid (moving outwards along the radial axis, $r$ ) and where the initial phases of the mixing process occur (for this reason this region is often called mixing layer). The velocity, temperature or density gradients along both sides of the shear layer will deeply affect its stability behavior. The disturbances present in the jet will trigger the formation of primary unstable KelvinHelmoltz structures that are amplified and grow until they saturate and roll up into discrete vortices undergoing several pairing and tearing processes until they finally collapse generating a complete turbulent flow. It is very well known that any shear flow may be characterized by varying size coherent structures, which are responsible for the energy exchange between the mean flow and the turbulence, especially in the near-field region of a jet. The evolution and interaction of theses turbulent structures create a complex three-dimensional flow field that eventually evolves to a self-similar state in the far-field. In spite of numerous experimental investigations of turbulent flows, many aspects of the jet flows remain unexplored because of the difficulty of accurately predicting the interaction of flow structures.

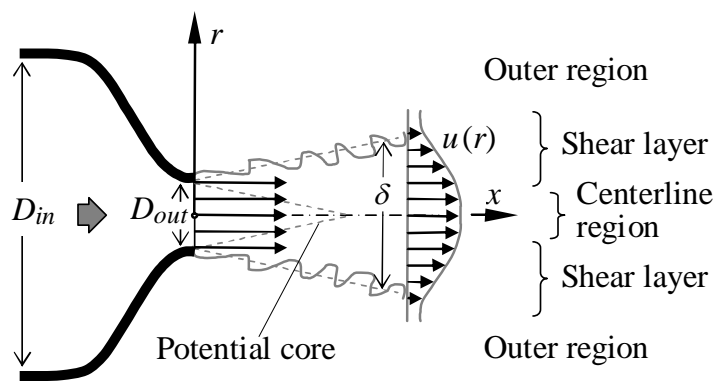

Figure 1. Elements of an axisymmetric free jet (adapted from Fellouah et al. (2009).

In this work, a qualitative as well as quantitative study of vortex formation in incompressible axisymmetric free jet for moderated Reynolds numbers less than 20,000 has been carried out utilizing hot-film anemometry and flow visualization.

\section{INCOMPRESSIBLE FREE JETS}

A free jet may be defined as a kinetic energy driven unobstructed and unrestricted flow of a fluid into a quiescent (or much slower) environment. Since a fluid boundary cannot sustain a pressure difference across it, the subsonic jet boundary is a free shear layer in which the static pressure is constant throughout. The boundary layer at the exit of the nozzle develops as a free shear layer, mixing with the ambient fluid thereby the ambient fluid entraining the jet stream. Thus, the mass flow at any cross section of the jet progressively increases, spreading the jet as it goes along the downstream direction. In order to conserve momentum, the jet centerline velocity decreases with downstream distance from the nozzle lip. As the buoyancy forces would have a role in the trajectory of jets, in the present work the jet is considered as non confined isothermal jet discharging into a large volume.

Several experimental investigations have shown that the incompressible axisymmetric free jet has a regular vortex structure - Popiel and Trass (1991) and Del Rio et al. (2012). Initially, instability waves start in the laminar shear layer and are amplified and rolled up into a train of discrete toroidal vortices, developing and convecting downstream along the shear layer surrounding a conical jet core - Fig. 2 . The initial flow conditions determine the momentum boundary layer thickness of the shear layer at the nozzle mouth, severely influencing the primary vortex structures and, consequently, the shear layer development and the vortex frequency. Consider $U o$ the mean velocity in the jet root or in the nozzle lip. The design of the contraction nozzle geometry should be carefully carried out in order to promote a very flat velocity profile with a low turbulence level and, additionally, keeping a thin laminar boundary layer along the contraction internal walls. Several works describe many different techniques for the aerodynamic contraction design. A review of a number of different axisymmetric contraction geometries design is available in the work of Vieira (1997).

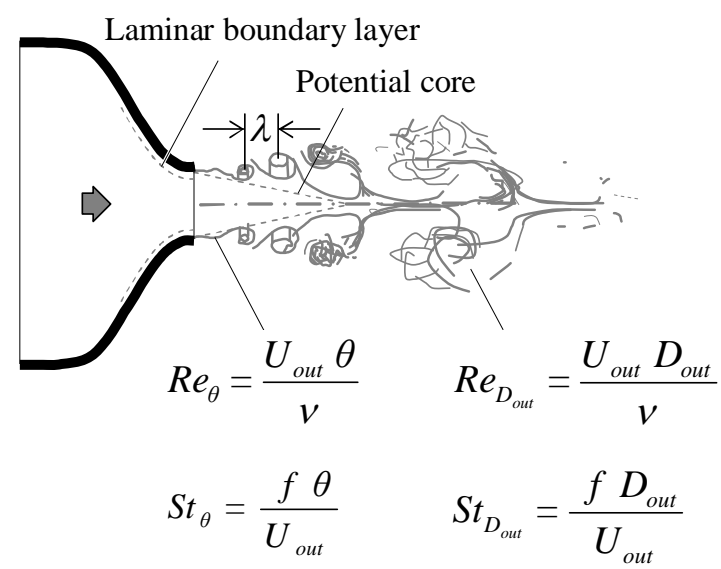

Figure 2. Typical vortex structure of an axisymmetric free jet - adapted from Popiel and Trass (1991).

The developing region of the round free jet has been studied by many experimenters, but only a few of them have presented flow visualization pictures, and these are usually of low resolution because visualization of axisymmetric shear layers and, consequently, round jets are difficult to achieve Popiel and Trass (1991). The need for visual study of 
jets arises from the serious discrepancies between theoretical results and heat transfer measurements in several tested cases - Gordon and Akfirat (1965). Very good quality images have been captured by Yokobori et al (1997) utilizing the hydrogen bubble technique. The flow visualized image analysis shows the flow behavior of a submerged round free jet. It was generally concluded, for impinging round jets that beyond the jet core length the mechanism of the stagnation heat transfer must be substantially affected by the large three-dimensional structures that are closely related to the vortex shear layer development upstream. Hydrogen bubble technique employs as tracing particles the small hydrogen bubbles produced by hydrolysis along a fine wire operating as a cathode. Unfortunately, the density gradient between the gas bubbles and the water produces an appreciable buoyancy force and bubble rising. In order to reduce the buoyancy effect the bubble diameter must be really small. However very tiny bubbles produce poor contrast and high-noise images. The use of hydrogen bubble technique for flow visualization is therefore restricted to flow velocities higher enough to turn buoyancy negligible in relationship to the dynamic forces. Thus for low Reynolds numbers broad dispersion of solid microparticles or injection of high-contrast liquid dye is deployed, in order to be able to reveal the shear layer details. An extensive review of flow visualization techniques as applied to hydrodynamic flow is available in Vieira (1997).

\section{EXPERIMENTAL APPARATUS}

Jets have been investigated using the three ways of scientific exploration: theoretical, computational simulation or modeling and experimental. In order to understand jet flows several experimental techniques can be utilized and that kind of flow has been object of analysis by many experimenters. However, in the case of impinging jets serious discrepancies between theoretical and heat transfer measurements results still remain as appointed by Popiel and Trass (1991). On the other hand, interest in experimental jet flow visualization has constantly grown in the last three decades. Several aspects related to the lasting effects on the jet by the root initial velocity and turbulence profiles remains unknown. Of course, turbulence and velocity profiles in the root of the jet severely influence the formation of turbulent large scale structures.

It cannot be stressed enough that the flow depicted in Fig. 2 only exists in a jet issuing from a very well designed convergent nozzle, i.e., the nozzle geometry should be carefully designed in order to produce a uniform initial velocity profile with a low turbulence level. Free jet issuing from a long pipe or from a sharp-edge orifice exhibits a highly turbulent non uniform velocity profile and just a residual form of a regular flow structure is observed - Popiel and
Trass (1991). The nozzle geometry determines the initial velocity distribution (Zuckerman and Lior, 2006). The velocity profile and turbulence level in the jet root determine the momentum thickness of the shear layer near the nozzle mouth producing a severe influence on the primary vortex structure and frequency. The velocity gradients in the jet create a shearing at the edges of the jet which transfers momentum laterally inwards, pulling additional fluid along with the jet and raising the jet mass flow, as shown in Fig. 2. In the process, the jet loses kinetic energy and the velocity profile is widened in spatial extent and decreased in magnitude along the sides of the jet.

The experimental apparatus utilized in this present work is described in details by Del Rio et al. (2012) and depicted in Fig. 3. This equipment is very similar to a small water tunnel with open test section. Previously, this apparatus had been intensely utilized to calibrate hot-film probes using a free jet with an initial low turbulence uniform velocity profile. Nowadays, numerous experimental studies of free and impinging jet in moderate Reynolds numbers less than $2 \times 10^{3}$ are additionally carried out utilizing exactly the same device.

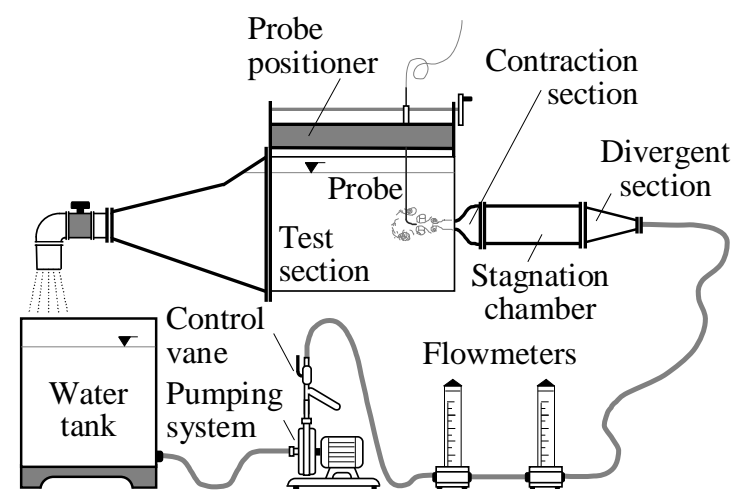

Figure 3. Experimental apparatus.

\section{RESULTS AND DISCUSSION}

Figure 4(a) depicts a typical hot-film probe calibration curve using the experimental apparatus described. It is important to stress the need of periodic recalibration of the hot-film probes because of the frequent loss of calibration while operating in water. In only one typical day of tests, three or more repeated calibration operations are absolutely necessary. Brunn (1995) relates many different techniques for hot-film probe calibration, but in the method utilized in the present work there is no need for the transfer of the probes between the calibrator device and the experimental apparatus. Figure 4(b) shows an example of the instantaneous velocity measured in the root of the jet. In the mouth of the contraction section the velocity fluctuations are very small owing to accurate design of the contraction 
shape and turbulence manipulators devices, such as honeycombs and screens, adequately positioned inside the stagnation chamber. A sample of 131,072 points of measurements with a sample frequency of $16 \mathrm{kHz}$ allows an acquisition time of $8.192 \mathrm{~s}$. The relative turbulence level obtained in the contraction mouth is less than $0.6 \%$ for the more adverse situation.

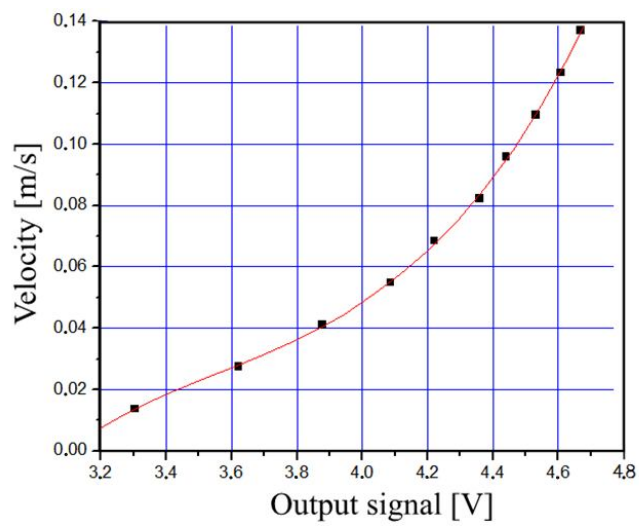

(a) Hot-film probe calibration curve.

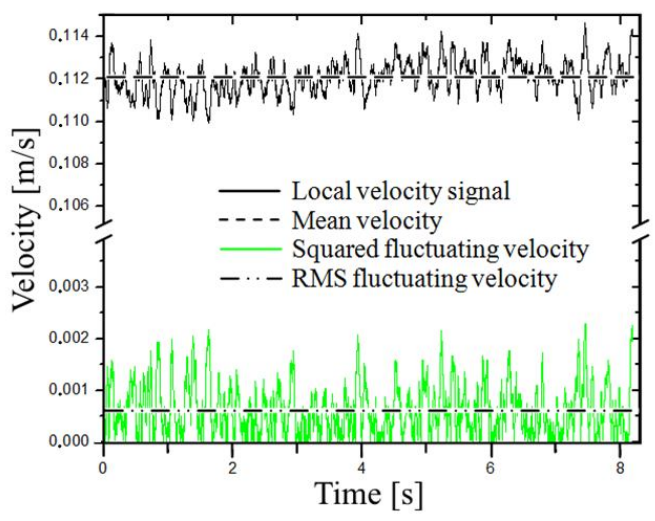

(b) Mean velocity and temporal fluctuation.

Figure 4. Treatment of data obtained by hot-film anemometry at the jet root.

Measurements of mean velocity and the turbulent level have been carried out in several radial positions inside the jet for a longitudinal length of one diameter $\left(1 D_{\text {out }}\right)$ from the jet root as shown in Fig. 5. Around the center of the jet it is possible to visualize a flat velocity profile with a near constant velocity. In this region internal the jet, a potential core (depicted in Fig. 1) is clearly observed. Inside the potential core the mean velocity is almost constant in the radial direction and the turbulence level remains very small, but, close the shear region a rise in the turbulence is observed. In the shear region, the mean velocity decrease and the turbulence increase in the radial direction. Outside the jet, the velocity is very small, but due to thermal convection in the hotfilm probe a residual value of velocity is detected.

In the Fig. 6 for a longitudinal length of $3 D_{\text {out }}$ from the jet root, the potential core exhibits a much smaller diameter. Finally, Fig. 7 for a longitudinal length of $5 D_{\text {out }}$ the potential core disappears, while the turbulence level always increases with the length from the jet root. Measurements of the mean temporal velocity and the mean turbulent level in the center of the jet with the longitudinal length from the

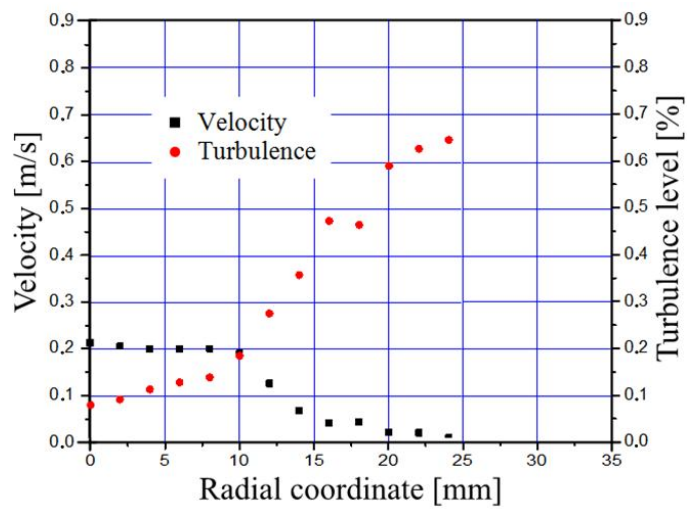

(a) $\operatorname{Re}=7,055$.

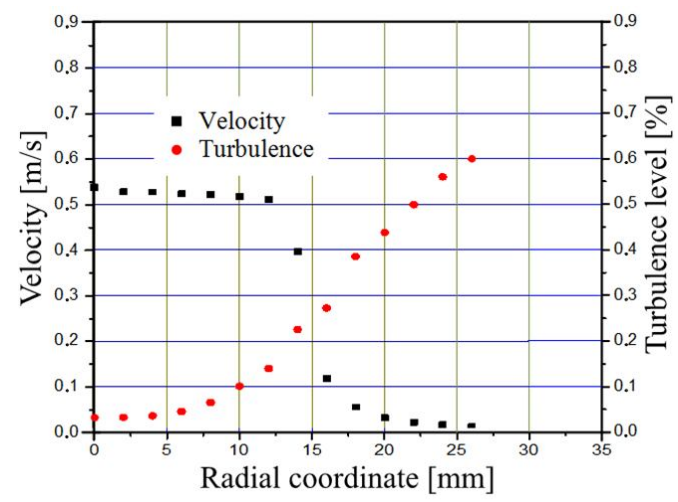

(b) $R e=17,418$.

Figure 5. Velocity and turbulence level profiles at $x=1 D_{\text {out }}$, and different Reynolds numbers.

jet root are depicted in Fig. 8. Finalizing, Fig. 9 shows some visualized images of the shear layer of the axisymmetric free jet, using liquid dye injection for two different Reynolds numbers. In this case is possible to visualize the shear layer and the vortex formation. The visualized images of the shear region depicted in Fig. 9 easily allow the identification the process of the vortex formation. Very close the jet root, the perturbations in the shear region appears very small. An increase of the perturbation is observed for the jet travelling farther the mouth of the injector. Then the collapse of the perturbations gives rise to the toroidal vortex structures as expected. 
In Fig. 10 the flow has been seed with Pliolite ${ }^{\circledR}$ micro particles and illuminated by a laser sheet produced by a biconvex lens. The laser scattering in the micro particles allows visualizing the jet flow in Reynolds number of 3,200. Several particle concentrations have been tested. An image obtained using low particle concentration Fig. 10(a) - is compared with other image using low concentration - Fig. 10(b). In both the images it is possible to visualize the process of formation and dissipation of the vortex.

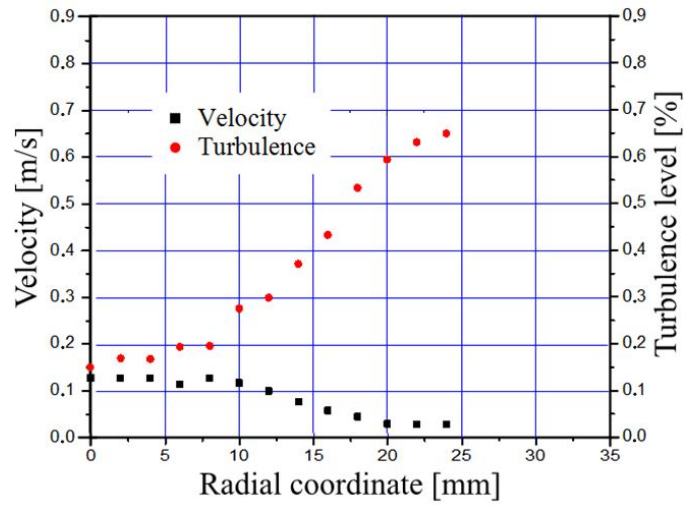

(a) $\operatorname{Re}=7,055$.

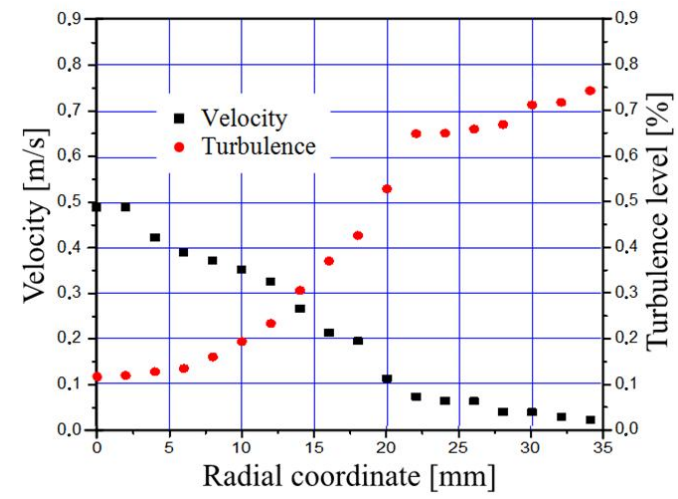

(b) $\operatorname{Re}=17,418$.

Figure 6. Velocity and turbulence level profiles at $x=3 D_{\text {out }}$, and different Reynolds numbers.

\section{CONCLUSION}

Jet cooling offers great potential for new important applications, in special to electronic components, due to the size reducing and the corresponding increasing in thermal dissipation needs. Several other new applications are possible to jet flows. The present research is a part of a series of investigations aimed at understanding the free jet flow behavior. Over the past decades, jet flows have been the subject of extensive experimental and analytical research. In spite of the research literature available on turbulent circular jets, many important aspects still require attention. In this work, hydrodynamic flow visualization and hot-film measurements have been performed to obtain qualitative and quantitative information about the vortex formation in a free jet. Vortex shedding phenomenon has been qualitatively visualized by means of still images. For moderate and low Reynolds numbers, the correct probe positioning is a very important factor to obtain a good noise to signal ratio. In this sense, flow visualization is an excellent tool, helping in the tedious work of adequately

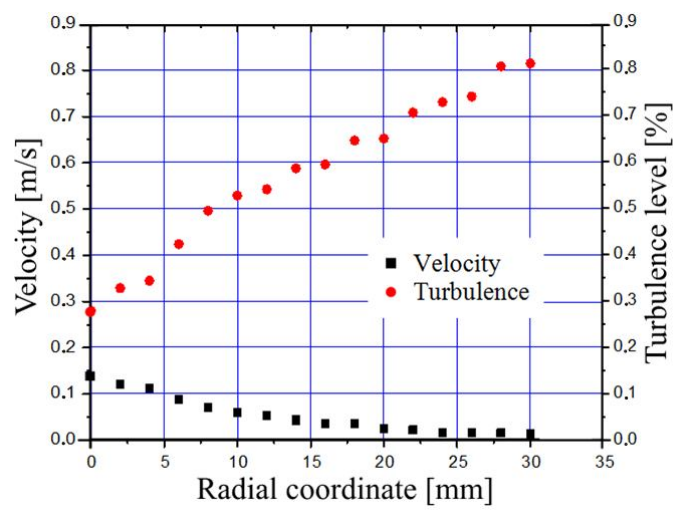

(a) $\operatorname{Re}=10,458$.

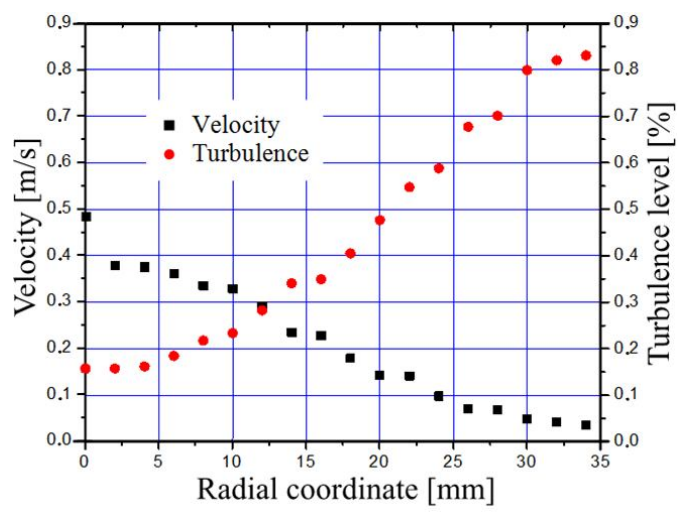

(b) $\operatorname{Re}=17,418$.

Figure 7. Velocity and turbulence level profiles at $x=5 D_{\text {out }}$, and different Reynolds numbers.

placing the hot-film probes in the flow. From the contrast obtained with the injection of liquid colored dye the vortices can be easily visualized.

In the study of jet flow the use of experimental measurements associated to flow visualization techniques has been highly recommended - Freymuth et al. (1983). The operation and limitations of hotwire anemometry are extensively related in several classical textbooks, like those by Perry (1982), Lomas (1986) or Bruun (1995) or more recently as that given in Tropea et al. (2007). But, these text books pay little attention to operation of hot-film anemometry. Several studies of free jets can be found in the technical literature, but only a few of them have presented flow visualization pictures, and those 
were usually of low resolution.

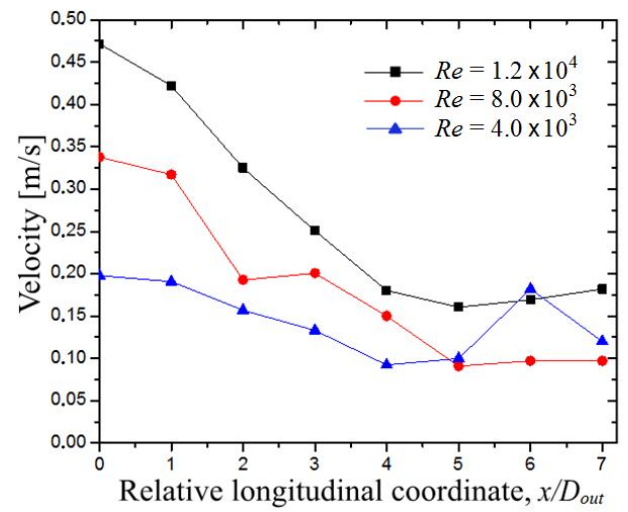

(a) Mean velocity.

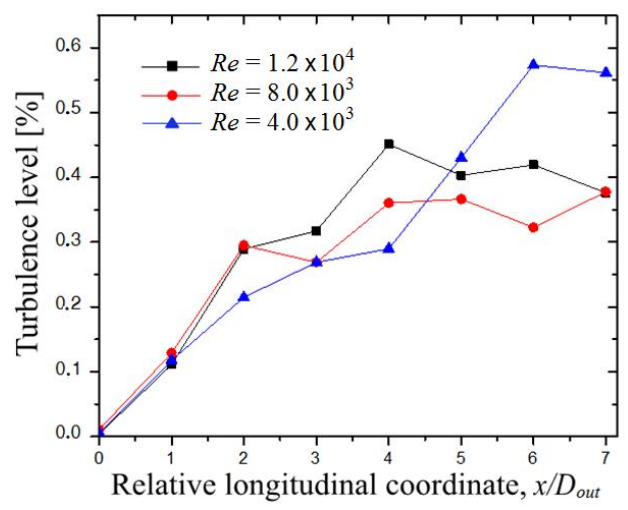

(b) Turbulence level.

Figure 8 . Temporal mean velocity and turbulence level along the jet centerline $(r=0)$.

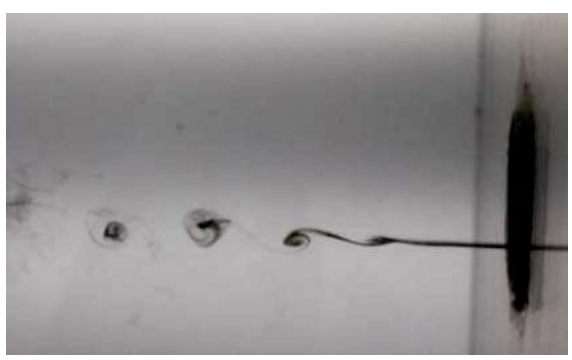

(a) $\operatorname{Re}=2,128$.

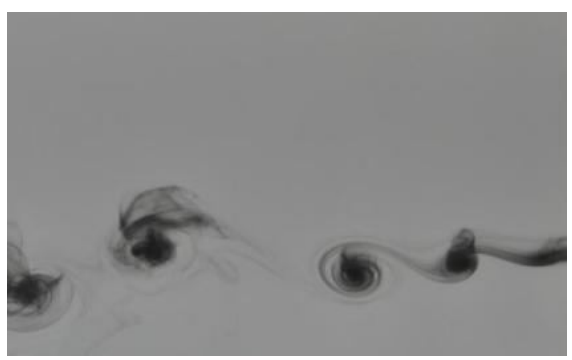

(b) $R e=7,055$.

Figure 9. Free jet shear layer flow visualization: dye injection technique.

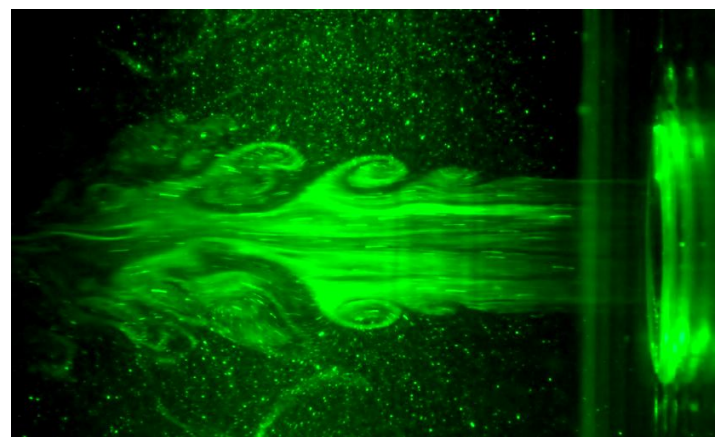

(a) Low particle concentration.

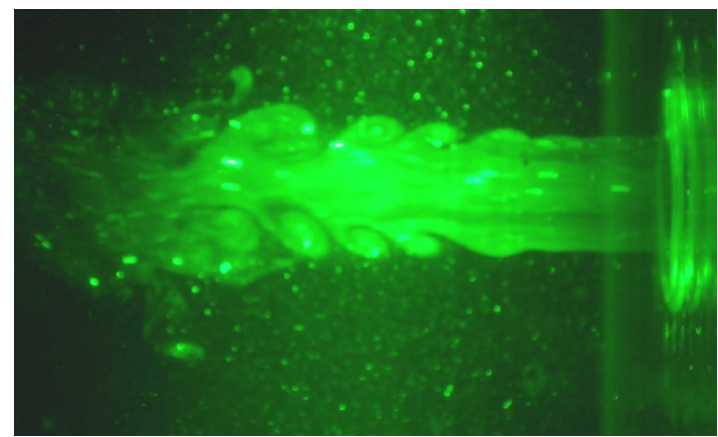

(b) High particle concentration.

Figure 10. Free jet flow visualization at $R e=3,200$ : micro particles and laser sheet technique.

\section{ACKNOWLEDGEMENTS}

The authors gratefully acknowledge financial support received from FAPESP and FUNDUNESP.

\section{REFERENCES}

Bruum, H. H., 1995, Hot-wire Anemometry, Oxford University Press, Oxford.

Del Rio, E. R. V, Vieira, E. D. R., and Mansur, S. S., 2012, Hot Film Probe Calibration Using a Free Jet, Proc. of the 8th Spring School on Transition and Turbulence - EPTT 2012, São Paulo, SP, Brazil.

Fellouah, H., Ball, C. G., and Pollard, A., 2009, Reynolds Number Effects within the Development Region of a Turbulent Free Jet, International Journal of Heat and Mass Transfer, Vol. 52, pp. 3943-3954.

Freymuth, P., Bank, W., and Palmer, M., 1983, Flow Visualization and Hot-wire Anemometry, TSI Quartely, Vol. 9, pp. 11-14.

Gordon, R. and Akfirat, J. C., 1965, The Role of Turbulence in Determining the Heat Transfer Characteristics of Impinging Jets, International Journal of Heat and Mass Transfer, Vol. 8, pp. 1261-1272.

Lomas, C. G., 1986, Fundamentals of the Hot-wire Anemometry, Cambridge University Press, Cambridge.

Pai, S.-I., 1954, Fluid Dynamics of Jets, D. Van Nostrand Company, Inc., London. 
Perry, A. E., 1982, Hot-wire Anemometry, Claremndon Press, Oxford.

Popiel, C. O, and Trass, O., 1991, Visualization of a Free and Impinging Round Jet, Experimental Thermal and Fluid Science, Vol. 4, pp. 253-264.

Schlichting, H., 1968, Boundary Layer Theory, Sixth edition, MacGraw Hill, New York.

Tropea, C., Yarin, A., and Foss, J., 2007, Springer Handbook of Experimental Fluid Mechanics, Springer-Verlag Berlin Heidelberg.

Vieira, E. D. R., 1997, Qualitative and Quantitative Study of the Flow Around Bluff Bodies Utilizing Hydrodynamic Flow Visualization Techniques, Doctoral Thesis, Instituto Tecnológico de Aeronáutica - ITA, São José dos Campos.

Yarin, L. P., 2012, The Pi-theorem: Applications to Fluid Mechanics and Heat and Mass Transfer, Spring-Verlag Berlin Heidelberg.

Yokobori, S., Kasagi, N., Hirata, M., Nakamura, M., and Haramura, Y., 1997, Characteristic Behavior of Turbulence and Transport Phenomena at the Stagnation Region of an Axisymmetrical Impinging Jet, in: Proc. of the 2nd International Symposium on Turbulent Shear Flows, London.

Zuckerman, N., and Lior, N., 2006, Jet Impingement Heat Transfer: Physics, Correlations, and Numerical Modeling, Advances in Heat Transfer, Vol. 39, pp. 565-631. 